\title{
An investigation of the use of the Hedin-Lundqvist exchange and correlation potential in EXAFS data analysis
}

\section{Mervyn Roy and S. J. Gurman}




\section{An investigation of the use of the Hedin-Lundqvist exchange and correlation potential in EXAFS data analysis}

\author{
Mervyn Roy* and S. J. Gurman \\ Department of Physics and Astronomy, University of Leicester, \\ University Road, Leicester, UK. E-mail:mr6@le.ac.uk
}

In real systems, inelastic processes remove photoelectrons from the elastic scattering channel. This reduces the amplitude of the EXAFS. Traditionally the discrepancies between experimental and theoretical amplitudes were treated by including two semi-empirical reduction factors in the data analysis. Some inelastic effects may, however, be modelled more rigorously using a complex exchange and correlation potential, for example the Hedin-Lundqvist (HL) potential used in most EXAFS data-analysis programs. In this paper a systematic study of the effects of the HL potential on the calculated EXAFS amplitudes is presented. Expressions are derived whereby the EXAFS amplitudes may be examined in the presence of an arbitrary complex potential independently to the rest of the EXAFS signal. These results are used to study the effects of the HL potential on EXAFS data analysis in detail.

Keywords: EXAFS; inelastic processes; exchange and correlation potential; EXAFS data analysis.

\section{Introduction}

The extended X-ray absorption fine structure, or EXAFS, is the fine structure observed in the X-ray absorption coefficient as a function of energy. This fine structure, beginning immediately above the edge and extending up to $1000 \mathrm{~V}$ or more beyond an absorption edge, contains information on atomic arrangements, bond lengths and coordination numbers.

Interpretation of the EXAFS may be based on a simple equation (Lee \& Pendry, 1975) obtained using the plane wave approximation. The single-electron single-scattering expression for the EXAFS function, $\chi(k)$, is,

$$
\chi_{i}(k)=\sum_{j} s_{o}^{2} e^{-2 r_{j} / \lambda} \frac{N_{j}\left|f_{j}(k, \pi)\right|}{k r_{j}^{2}} \sin \left[2 k r_{j}+2 \delta_{j}(k, r)+\psi_{j}\right] .
$$

Equation (1) describes the EXAFS due to scattering by shells of $N_{j}$ atoms at a distance $r_{j}$ from the absorbing atom. $f_{j}(k)$ is the backscattering amplitude from each of the $N_{j}$ neighbours whilst the reduction factors $e^{-2 r_{j} / \lambda}$ and $s_{o}^{2}$ account for discrepancies between the predicted and experimentally measured amplitudes.

These discrepancies arise because only elastically scattered photoelectrons contribute to the primary channel EXAFS. With a real scattering potential, the reduction factors are needed to account for effective absorptions from the photoelectron beam due to various inelastic scattering processes. The mean-free-path term, $e^{-2 r_{j} / \lambda}$, accounts for losses from inelastic scattering events at neighbouring atoms and in the medium in-between, whilst the constant amplitude reduction factor, $s_{o}^{2}$, otherwise known as AFAC, approximates the intrinsic losses, those due to multiple electron excitations at the absorbing atom. Traditionally, the factor $V_{P I}$ in the mean-free-path term, $\lambda=k / V_{P I}$, is approximated by the canonical value of $4 \mathrm{eV}$ given by LEED calculations whilst the constant amplitude reduction factor is taken to be $\sim 0.7$.
Most modern EXAFS data-analysis programs use a complex exchange and correlation potential to model inelastic effects, for example the Hedin-Lundqvist (HL) potential (Hedin \& Lundqvist, 1969) used in the standard Daresbury package EXCURV 98 (Binsted, 1998 ) and in the equivalent American program, FEFF (Zabinsky et $a l ., 1995)$. This potential gives rise to complex phase shifts in equation (1) and in the more accurate curved-wave (Gurman et al., 1984) forms of the theory typically used in data analysis, and therefore includes some, if not all, of the reduction effects.

The HL potential is only designed to model the so-called extrinsic losses, those usually approximated by the mean-free-path term. In this case, EXAFS data analysis should be performed with a default value of AFAC of approximately 0.7 (see, for example EXCURV92). However, some years experience by EXAFS users at Daresbury demonstrated this value of AFAC to be too low. It was believed that the HL potential was overestimating the extrinsic losses and therefore, accidentally, including some of the weight of the intrinsic losses from multiple excitations at the absorbing atom. This led to the default value of AFAC being set to unity in the current issue of EXCURV (EXCURV98). The accuracy of the HL potential has been the subject of much discussion in the XAS community over recent years (Fujikawa et al., 2000; Newville et al., 1993; Tyson et al., 1992), although as yet we are not aware of any viable alternatives to its use for routine data analysis.

In this paper we present a systematic examination of the EXAFS amplitudes produced by the imaginary part of the HL potential. We demonstrate that this potential does indeed overestimate the extrinsic losses, and that the HL potential gives good agreement with the total losses from the elastically scattered photoelectron wave in the EXAFS range of photoelectron energies. This agreement is, however, somewhat fortuitous and we therefore conclude that, whilst the HL potential is good enough at present, some thought should be given to improving the modelling of inelastic processes in future issues of EXCURV and other data-analysis programs.

The paper is broadly split into two sections. In the first, we briefly detail the method used to calculate the EXAFS amplitude in the presence of the HL potential. In the second, we calculate the losses generated by the HL potential using this theory and compare them with the historically used EXAFS loss factors: a constant amplitude reduction factor, $s_{o}^{2}$, and a mean free path term, $e^{-2 r_{j} / \lambda}$.

This paper expands upon and clarifies a short note in the conference proceedings of XAFS X (Roy \& Gurman, 1999). In the final results obtained here we have used SI units of electron volts and Angströms. In the theory section, however, we use Hartree units for simplicity.

\section{Theory}

\subsection{The EXAFS amplitudes}

In principle we could use a program such as EXCURV98 to investigate the effects of the HL potential on the EXAFS amplitudes. The full EXAFS could be calculated both using the HL potential and using a real scattering potential such as the $\mathrm{X} \alpha$ potential, and the amplitudes compared. However, the two potentials give fine structure of a different phase which makes a direct comparison problematical. Instead we choose to calculate the effect of the imaginary part of the HL potential on the EXAFS in the first Born approximation. This gives the amplitude diminution directly.

Standard single electron EXAFS theory may be developed within the muffin tin approximation using the Hartree photoelectron Green function, $G_{o}\left(\mathbf{r}, \mathbf{r}^{\prime}\right)$, 


$$
\begin{aligned}
G_{o}\left(\mathbf{r}, \mathbf{r}^{\prime}, \omega\right)= & -l k \sum_{l m}\left(e^{\imath \delta_{l}} h_{l}^{(1)}\left(k r^{\prime}\right)+e^{-l \delta_{l}} h_{l}^{(2)}\left(k r^{\prime}\right)\right) \\
& \times h_{l}^{(1)}(k r) e^{l \delta_{l}} Y_{l m}^{*}(\hat{r}) Y_{l m}\left(\hat{r}^{\prime}\right), \quad r>r^{\prime}>r_{m t} \\
= & -l k \sum_{l m} h_{l}^{(1)}(k r) e^{l \delta_{l}} R_{l}\left(k r^{\prime}\right) Y_{l m}^{*}(\hat{r}) Y_{l m}\left(\hat{r}^{\prime}\right), \\
& r>r_{m t}>r^{\prime} \\
= & -l k \sum_{l m} R_{l}\left(k r^{\prime}\right) \frac{1}{2}\left(R_{l}(k r)-l X_{l}(k r)\right) \\
& \times Y_{l m}^{*}(\hat{r}) Y_{l m}\left(\hat{r}^{\prime}\right), \quad r_{m t}>r>r^{\prime},
\end{aligned}
$$

where $R_{l}(k r)$ and $X_{l}(k r)$ are the regular and irregular scattering solutions to the Schrödinger equation in the presence of the atomic potential whilst the free space wavefunctions have been defined in terms of the spherical Hankel functions, $h_{l}^{(1)}(k r)$ and $h_{l}^{(2)}(k r)$. The muffin tin radius is $r_{m t}, \omega=\frac{1}{2} k^{2}$, and we have taken the continuum scattering state wavefunctions to be

$$
\begin{gathered}
\psi(\mathbf{k}, \mathbf{r})=\sum_{l m} 2 \pi l l^{l} e^{i \delta_{l}}\left(e^{i \delta_{l}} h_{l}^{(1)}(k r)-e^{-i \delta_{l}} h_{l}^{(2)}(k r)\right) \\
\times Y_{l m}(\hat{r}) Y_{l m}^{*}(\hat{k}), \quad r>r_{m t}, \\
\psi(\mathbf{k}, \mathbf{r})=\sum_{l m} 2 \pi l e^{l \delta_{l}} R_{l}(k r) Y_{l m}(\hat{r}) Y_{l m}^{*}(\hat{k}), \quad r<r_{m t},
\end{gathered}
$$

where $\delta_{l}$ are the partial wave phase-shifts.

The extended X-ray absorption fine structure may be evaluated from the Green function in many different ways (Gurman et al., 1984). In this paper we use the Hartree Green function to examine the photoelectron flux. The photoelectron flux is directly related to the X-ray absorption coefficient and hence the EXAFS function by

$$
\mu(\omega)=\frac{8 \pi \omega S}{c E_{o}^{2}},
$$

where $S$ is the photoelectron flux through a sphere of radius $r$, centred on the absorbing atom, $\omega$ is the X-ray energy, and $c E_{o}^{2}$ is the rate of energy transport in the X-ray beam.

The photoelectron flux may be calculated in the usual fashion from the photoelectron wavefunction,

$$
\Psi=G_{c}\left(\mathbf{r}, \mathbf{r}^{\prime}\right) H^{\prime}\left(\mathbf{r}^{\prime}\right) \phi_{o}\left(\mathbf{r}^{\prime}\right) .
$$

In equation (6), we have chosen to examine the photoelectron wavefunction far from the absorbing atom, thus $r>r_{m t}>r^{\prime}$. $\phi_{o}(\mathbf{r})$, a well defined atomic initial state of energy $\omega_{o}$, is zero outside of the central atom muffin tin, the Green function is evaluated at an energy $\omega-\left|\omega_{o}\right|$ and, in the dipole approximation, the perturbation, $H^{\prime}\left(\mathbf{r}^{\prime}\right)$, due to the presence of the X-ray beam is simply $E_{o} \epsilon \cdot \mathbf{r}^{\prime}$.

In the presence of a single scattering atom, the relevant Green function, $G_{c}$, becomes

$$
G_{c}\left(\mathbf{r}, \mathbf{r}^{\prime}\right)=G_{o}\left(\mathbf{r}, \mathbf{r}^{\prime}\right)+G_{o}\left(\mathbf{r}, \mathbf{r}_{1}\right) T\left(\mathbf{r}_{1}, \mathbf{r}_{2}\right) G_{o}\left(\mathbf{r}_{2}, \mathbf{r}^{\prime}\right),
$$

where $T\left(\mathbf{r}_{1}, \mathbf{r}_{2}\right)$ is the T matrix of the scattering atom and $r$ is greater than $r_{1}$ and $r_{2}$ which are in turn larger than the muffin tin radius and hence $r^{\prime}$. Then, using standard results from the many derivations of the EXAFS function available, we find the photoelectron flux to be given by,

$$
S=\frac{k E_{o}^{2}}{6} \sum_{l} A\left(l, l_{o}\right)\left|\left\langle R_{l}|r| \phi_{l_{o}}\right\rangle\right|^{2}(1+\chi(k)) .
$$

$A\left(l, l_{o}\right)$ is the angle factor of Gurman (1983), whilst $\chi(k)$ is the EXAFS function of equation (1), or a more accurate equivalent. The
X-ray absorption coefficient from a single initial state of angular momentum $l_{o}$ is therefore given by

$$
\begin{aligned}
\mu(\omega) & =\frac{2 \pi k \omega}{3 c} \sum_{l} A\left(l, l_{o}\right)\left|\left\langle R_{l}|r| \phi_{l_{o}}\right\rangle\right|^{2}(1+\chi(k)) \\
& =\mu_{o}(\omega)(1+\chi(k)),
\end{aligned}
$$

where the sum over $l$ is over the possible angular momenta of the final state. This is the standard result for the X-ray absorption coefficient in the absence of inelastic scattering. Finally, the backscattering factor $f_{j}(k, \pi)$ from equation (1) is found to be

$$
f_{j}(k, \pi)=-\frac{2 \pi}{l k} \sum_{L}(-1)^{L}(2 L+1)\left(1-e^{2 \imath \delta_{L}}\right)=\left|f_{j}(k, \pi)\right| e^{\imath \psi} .
$$

In this paper we are interested in the EXAFS function in the presence of an arbitrary energy-dependent imaginary part of the potential. We treat this imaginary part as a small perturbation on the system. Then, to first order in the imaginary part of the potential, $V_{I}$, the Hartree Green function becomes,

$$
G_{1}\left(\mathbf{r}, \mathbf{r}^{\prime}\right)=G_{o}\left(\mathbf{r}, \mathbf{r}^{\prime}\right)-l G_{o}\left(\mathbf{r}, \mathbf{r}_{1}\right) V_{I}\left(r_{1}\right) G_{o}\left(\mathbf{r}_{1}, \mathbf{r}^{\prime}\right),
$$

where the Green function, $G_{o}\left(\mathbf{r}, \mathbf{r}^{\prime}\right)$, has $r^{\prime}<r_{m t}$ and $r>r_{m t}$ whilst $G_{o}\left(\mathbf{r}_{1}, \mathbf{r}^{\prime}\right)$ has both $r_{1}$ and $r^{\prime}$ inside the muffin tin. We assume that $r_{1}>r^{\prime}$ as the initial state, $\phi_{l_{o}}$, will always be highly localized whilst the imaginary part of the potential, $V_{I}$, is zero toward the centre of the atom (this shall be demonstrated in section 3). This means that the double integral in equation (6) will only be significant in the regions where $r_{1}>r^{\prime}$. Also, within the muffin tin approximation, $V_{I}$ is spherically symmetric so that the angular integrals over the directions of $\mathbf{r}_{1}$ simply reduce to the orthogonality integrals for the spherical harmonics.

The perturbed Green function always has the same functional form as $G_{o}$ whether or not $\mathbf{r}$ and $\mathbf{r}^{\prime}$ are inside or outside the muffin tin. For $\mathbf{r}$ and $\mathbf{r}^{\prime}$ outside the muffin tin the Green function will simply be multiplied by a numerical factor $\left(1-\frac{1}{2} k\left\langle R_{l}\left|V_{I}\right| R_{l}\right\rangle\right)$ whilst with either $\mathbf{r}$ or $\mathbf{r}^{\prime}$ or both inside the muffin tin the perturbed Green function can be written as $G_{o}\left(1-\frac{1}{2} k\left\langle R_{l}\left|V_{I}\right| R_{l}-\imath X_{l}\right\rangle\right)$. We shall see later that the ${ }_{l}\left\langle R_{l}\left|V_{I}\right| X_{l}\right\rangle$ term may be ignored. However, for the moment, including this term, we may rewrite equation (11) for the perturbed Green function as

$$
G_{1}\left(\mathbf{r}, \mathbf{r}^{\prime}\right)=-l k \sum_{l, m} e^{l \delta_{l}^{\prime}} h_{l}^{(1)}(k r) Y_{l m}^{*}(\hat{r}) R_{l}\left(k r^{\prime}\right) Y_{l m}\left(\hat{r}^{\prime}\right),
$$

where we have subsumed the factor $\left(1-\frac{k}{2}\left\langle R_{l}\left|V_{I}\right| R_{l}-\imath X_{l}\right\rangle\right)$ into the phase shifts making $\delta_{l}$ complex. In equation (12), $\delta_{l}^{\prime}$ is the perturbed phase shift. To first order,

$$
\delta_{l}^{\prime} \simeq \delta_{l}-\frac{1}{2}\left\langle R_{l}\left|V_{I}\right| R_{l}-{ }_{l} X_{l}\right\rangle .
$$

This is, of course, an approximate form for the perturbed phase shifts; however, using this form we can reproduce the textbook expression for the loss of flux from an isolated atom (Bransden \& Joachain, 1983). We therefore believe that this approximation is a good one.

Using result (13) for the perturbed phase shifts we may easily calculate the EXAFS in the presence of the imaginary potential by following the standard derivation of the EXAFS. From equation (1) we can see the effect on the EXAFS of perturbing the phase-shifts. We can also calculate the elastic contribution to the absorption coefficient. Writing the perturbed backscattering factor as $f_{j}^{\prime}(k, \pi)$ we have 


$$
\mu_{e l}=\mu_{o}\left(1-k\left\langle R_{l}\left|V_{I}\right| R_{l}\right\rangle\right)\left[1+2 R e \sum_{j} \frac{i(-1)^{l}}{2 k r_{j}^{2}} e^{2\left(k r_{j}+\delta_{l}^{\prime}\right.} f_{j}^{\prime}(k, \pi)\right] .
$$

The total absorption must be the same regardless of whether or not there is an imaginary part to the potential, as, in both cases, we must satisfy the Thomas-Reiche-Kohn sum rule. Thus, we may write the total X-ray absorption coefficient as,

$$
\mu_{\text {tot }}=\mu=\mu_{e l}(1+\chi)+\mu_{\text {inel }},
$$

which gives the EXAFS as

$$
\chi=\left(1-2 k\left\langle R_{l}\left|V_{I}\right| R_{l}\right\rangle\right) \frac{(-1)^{l}}{k r_{j}^{2}} \sin \left(2 k r_{j}+2 \delta_{l}+\psi\right)\left|f_{j}^{\prime}(k, \pi)\right| .
$$

The perturbed backscattering factor may be written as

$$
\begin{aligned}
f_{j}^{\prime}(k, \pi)= & \frac{i}{2 k} \sum_{L}\left[1-\left(1-k\left\langle R_{L}\left|V_{I}\right| R_{L}\right\rangle\right) e^{\imath\left(\delta_{L}-\left\langle R_{L}\left|V_{I}\right| X_{L}\right\rangle\right)}\right] \\
& \times(-1)^{L}(2 L+1) .
\end{aligned}
$$

This is a corrected version of the mis-printed equation (3) in Roy \& Gurman (1999). The irregular solution to the Schrödinger equation only appears in the exponential in the above equation. It merely alters the phase of the backscattering factor, $f_{j}^{\prime}(k, \pi)$. However, compared with the phase shifts, the radial matrix element $\left\langle R_{L}\left|V_{I}\right| X_{L}\right\rangle$ is always small. This is because the imaginary part of the HL potential, $V_{I}$, is approximately constant over much of the region of the atom. Thus, as $R_{L}$ and $X_{L}$ are orthogonal, the matrix element $\left\langle R_{L}\left|V_{I}\right| X_{L}\right\rangle$ must be small. From Fig. 1 we can see that for most of the energy range investigated $\left\langle R_{l}\left|V_{I}\right| X_{l}\right\rangle$ is much less than $1 \%$ of the phase shifts. The neglect of this term in the calculation of the EXAFS amplitude is therefore valid.

Thus, in the presence of an imaginary part to the potential the EXAFS amplitude is given to first order in $V_{I}$ by

$$
A_{l}=1-2 k\left\langle R_{l}\left|V_{I}\right| R_{l}\right\rangle\left|\frac{f_{j}^{\prime}(\pi)}{f_{j}(\pi)}\right| .
$$

In the above equation we can identify two parts to the amplitude reduction: $2 k\left\langle R_{l}\left|V_{I}\right| R_{l}\right\rangle$ comes from the central atom muffin tin; $k\left\langle R_{l}\left|V_{I}\right| R_{l}\right\rangle$ is the flux lost from the elastically scattered photoelectron wave originating from an isolated atom. In EXAFS, of course,

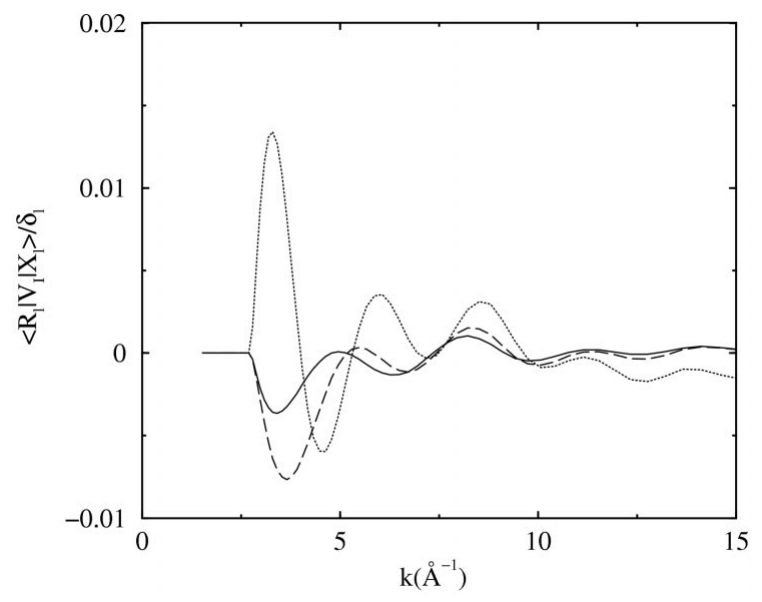

Figure 1

The matrix element $\left\langle R_{l}\left|V_{I}\right| X_{l}\right\rangle$ divided by the phase shift $\delta_{l}$ at $l=0$ (solid line), $l=1$ (dashed line) and $l=2$ (dotted line). the photoelectron wave encounters the central atom potential twice, once on the way out and once on the way back in. The $\left|f_{j}^{\prime}(\pi) / f_{j}(\pi)\right|$ term is the reduction to the EXAFS amplitudes caused by inelastic excitations at the scattering atoms.

\subsection{The $H L$ potential}

The HL potential was originally developed to model exchange and correlation effects in a uniform electron gas (Hedin \& Lundqvist, 1969). This model goes beyond the Hartree Fock theory by using a single plasmon pole (SPP) form of the dielectric function to calculate the electron self energy. The SPP dielectric function approximates all the inelastic electron-electron interactions as plasmons. The weight of the plasmon absorptions is adjusted so as to approximate the total of the actual plasmon excitations plus particle-hole excitations. Tyson et al. (1992) argue that this adjustment of the weight of the plasmon excitations should lead to good results for the total (plasmon plus single particle) extrinsic inelastic excitations. It does not include the intrinsic effects due to the appearance of the core hole. This may easily be seen since the HL losses go to zero at very high photoelectron energies whereas the core hole effects tend to a constant loss in this limit [see discussion of equation (22) below].

The HL potential was first applied to EXAFS calculations within the local density approximation by Lee \& Beni (1976). Its calculation is a standard problem. Here we simply quote the result for the imaginary part of the potential,

$$
V_{I}\left(r, E_{i}\right)=-\frac{\omega_{p}^{2}}{2 k} \int_{o}^{q_{\max }} \frac{d q}{q \omega_{q}} \int_{-1}^{x_{1}} \delta\left(x-\frac{\frac{1}{2} q^{2}+\omega_{q}}{k q}\right) d x,
$$

where $E_{i}=\omega-\left|\omega_{o}\right|$ is the photoelectron energy, $\omega_{p}$ is the plasma frequency of an electron gas of density $n, \omega_{p}^{2}=4 \pi n$. In the local density approximation both the electron density and hence $\omega_{p}$ depend on $r$. The $q$-dependent plasmon excitation frequency is $\omega_{q}^{2}=\omega_{p}^{2}+\frac{1}{3} q^{2} k_{f}^{2}+\frac{1}{4} q^{4}$, and $q_{\max }=k+k_{f}$ where $\frac{1}{2} k_{f}^{2}=V_{o}(r)+E_{f}$ is the kinetic energy at the Fermi level. $V_{o}(r)$ is the atomic potential and $E_{f}$ is the thermodynamic Fermi energy. The imaginary part of the exchange and correlation potential is only non-zero for electrons with energies greater than that needed to excite a local plasmon, $E_{i}-E_{f}>\omega_{q}$. Below this energy the incident electrons are not energetic enough to excite a plasmon and so, in the single plasmon pole approximation, inelastic scattering events are impossible.

\section{Results}

In this section we use equation (18) to examine the effects of the HL potential on the EXAFS amplitudes for a number of different elements. Primarily we look at silicon, copper and silver as experimental data is available for these three elements, which cover a wide range of atomic number and $K$-edge energies. Fig. 2 shows the imaginary part of the HL potential calculated for silicon, copper and silver.

For all elements, the imaginary part of the HL potential is zero at small $r$. In this region of high electronic density the incident photoelectron is insufficiently energetic to excite a plasmon. As soon as the density falls sufficiently for plasmons to be excited, $V_{I}$ cuts in almost immediately to its maximum value. At larger incident photoelectron energies, $V_{I}$ obviously cuts in at lower radii; however, for large $E_{i}$ the imaginary part of the potential is generally smaller in the outer regions of the atomic muffin tins. The energy dependence of the imaginary part of the HL potential is shown for bromine in Fig. 1 of Lee \& Beni (1976). 
The imaginary part of the HL potential looks very similar for the three very different elements: silicon, copper and silver. The electron mean free paths for these elements will therefore also be very similar as has long been found experimentally.

The radius at which the HL potential cuts in moves slightly outward as we go up in atomic number. This is because the electron density is larger close to the nucleus for elements with higher atomic numbers. The corresponding plasmon excitation frequency is therefore higher and so we have to move further from the centre of the atom before it becomes possible to excite a plasmon with a photoelectron of given energy.

It is instructive to examine the average imaginary part of the HL potential. This can be calculated simply from,

$$
V_{A V G}(k)=\frac{3}{r_{m t}^{3}} \int_{o}^{r_{m t}} V_{I}(r, k) r^{2} d r .
$$

In Fig. 3 we plot the average imaginary part of the potential against $k$ for copper. The imaginary part of the potential has an average value of approximately $4.8 \mathrm{eV}$ over most of the range of energy appropriate to EXAFS data analysis. This is close to, but slightly higher than, the

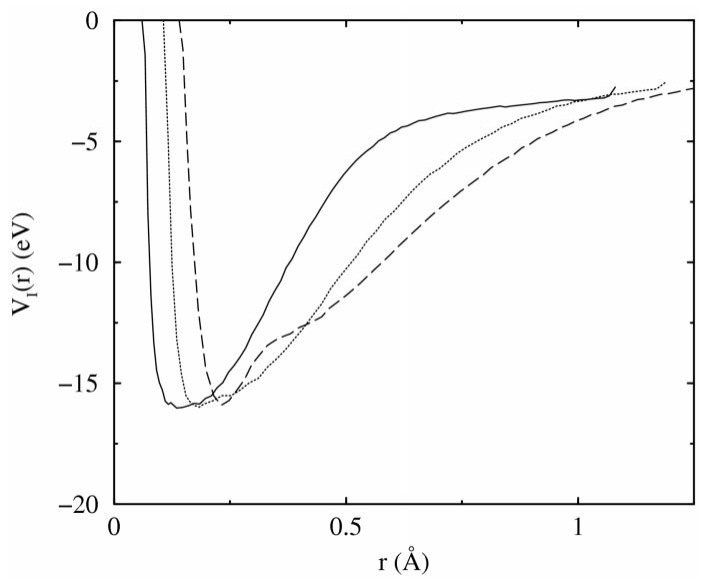

Figure 2

The imaginary part of the HL potential in $\mathrm{eV}$, at $k=15 \AA^{-1}$. Calculated for silicon (solid line), copper (dotted line) and silver (dashed line).

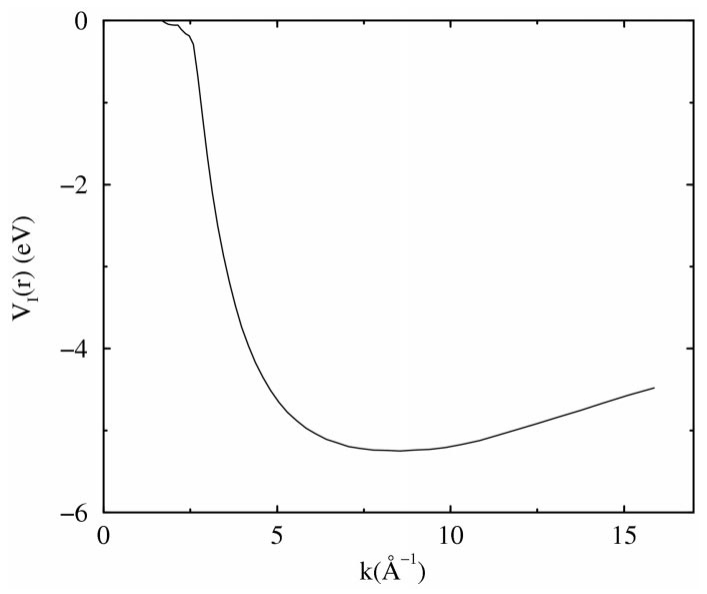

Figure 3

The average imaginary part of the HL potential plotted as a function of $k$ for copper. canonical value of $4 \mathrm{eV}$ generally used to account for the extrinsic losses in LEED calculations for example (Pendry, 1974). The average imaginary part of the HL potential is also very similar for all atoms. This is a consequence of describing the atoms as shells of varying electron density.

Fig. 3 also shows that the average potential falls off with increasing photon energy above the edge. This can also be deduced from equation (19). We only obtain a contribution from the delta function if,

$$
\left|\frac{\frac{1}{2} q^{2}+\omega_{q}}{k q}\right|<1
$$

Taking the limit of high photoelectron energy so that $k$ becomes very large and $\frac{1}{2} k^{2}>>\omega_{p}$ we find that we will always obtain a contribution from the delta function provided that $q<k$ and $q>\omega_{p} / k$. Then, at high $k$,

$$
V_{I}=\frac{\omega_{p}^{2}}{2} \int_{\frac{\omega_{p}}{k}}^{k} \frac{d q}{k q \omega_{q}}=\frac{\omega_{p}}{k}\left[\frac{1}{2} \log \left(\frac{k q_{c}}{\omega_{p}}\right)+\frac{\omega_{p}}{3 q_{c}^{2}}\right],
$$

where, to a first approximation, we have approximated $\omega_{q}$ with its low $q$ limit (of $\omega_{p}$ ) below $q_{c}$ and with its high $q$ limit of $\frac{1}{2} q^{2}$ above $q_{c}$. We can see that equation (22) for $V_{I}$ will obviously go to zero as $k$ becomes very large. As $V_{I}$ is designed to model only the extrinsic photoelectron scatterings this is correct.

In Figs. 4(a) and 4(b) we compare the measured $k^{3}$ weighted EXAFS for crystalline silicon to best-fit theoretical curves calculated with $(a)$ the $\mathrm{X} \alpha$ potential including an amplitude reduction factor of
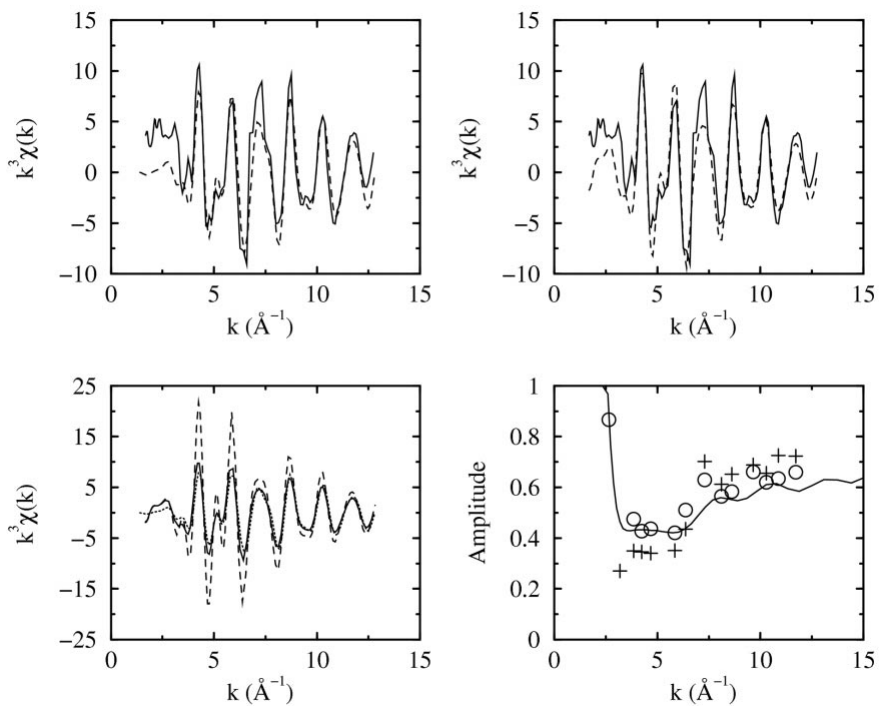

Figure 4

Clockwise from the top left: (a) A comparison between the measured $k^{3}$ weighted EXAFS for crystalline silicon (solid line) and a fitted EXAFS spectrum calculated using the $\mathrm{X} \alpha$ potential with correction factors (dashed line). (b) A comparison between the experimental silicon spectrum (solid line) and the EXAFS calculated using the HL potential with no additional correction factors (dashed line). (c) The two calculated EXAFS spectra from above shown against the EXAFS calculated using the X $\alpha$ potential with no correction factors (dashed line). The HL potential result is the solid line and the $\mathrm{X} \alpha$ result with an amplitude correction is the dotted line. (d) The approximate result for the EXAFS amplitude calculated using equation (18) compared with a result obtained by taking the ratio of the amplitudes of equivalent peaks from the EXAFS plotted in (c) calculated using the HL potential and the $\mathrm{X} \alpha$ potential with no correction factors (circles). The crosses show the same ratio for the EXAFS calculated using the $\mathrm{X} \alpha$ potential with and without correction factors. 
Table 1

Interatomic distances and Debye-Waller factors.

\begin{tabular}{lrllcc}
\hline Shell & $\mathrm{N}$ & $R_{H L}(\AA)$ & $R_{X \alpha}(\AA)$ & $\sigma_{H L}^{2}\left(\times 10^{-4}\right)$ & $\sigma_{X \alpha}^{2}\left(\times 10^{-4}\right)$ \\
\hline 1 & 4 & $2.36 \pm 0.02$ & $2.34 \pm 0.02$ & $45 \pm 15$ & $45 \pm 10$ \\
2 & 12 & $3.86 \pm 0.05$ & $3.84 \pm 0.05$ & $135 \pm 50$ & $130 \pm 40$ \\
3 & 12 & $4.50 \pm 0.05$ & $4.52 \pm 0.05$ & $140 \pm 80$ & $200 \pm 50$ \\
\hline
\end{tabular}

$0.7 \pm 0.1$ and a constant imaginary potential of $-4 \pm 0.2 \mathrm{eV}$, and $(b)$ the HL potential. Here the mean free path $\lambda(k)=k / V_{P I}$ so that, with a constant imaginary potential, $V_{P I}$, the mean free path varies as $\sqrt{E}$ as usual. In reality $s_{o}^{2}$ will obviously have some energy dependence (Roy et al., 2001), but in this traditional method of data analysis $s_{o}^{2}$ is always approximated by its constant high-energy limit.

The silicon spectrum [published by Gurman \& McGreevy (1990)] was measured on beamline 3.4 of the CLRC Daresbury laboratory. Pre- and post-edge background was subtracted using polynomial fits (EXBACK) and the background-subtracted spectra fitted using EXCURV 98 using three shells with fixed coordination number. The interatomic distances and Debye-Waller factors obtained are shown in Table 1. The fit using the $\mathrm{X} \alpha$ potential gives a fit index of 39.2. The HL potential gives a slightly better match to experiment, with a fit

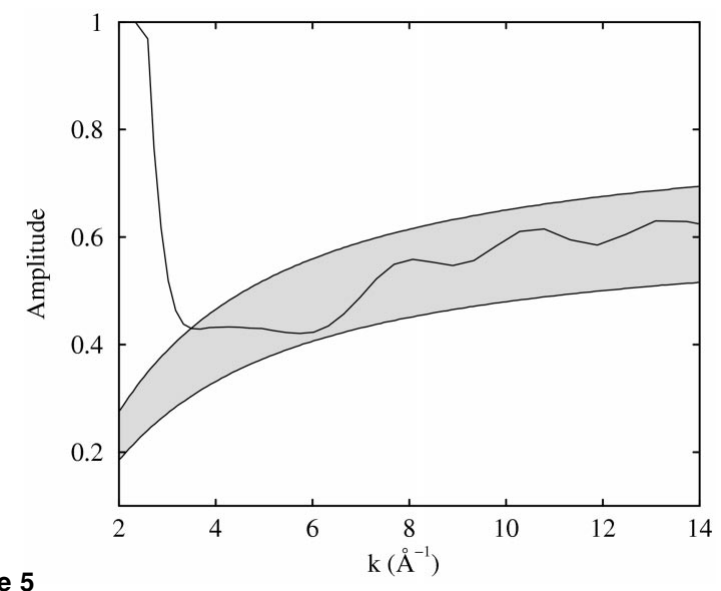

Figure 5

Calculated and best-fit EXAFS reduction factors plotted against photoelectron wavevector for silicon. The black line gives the calculated result, the solid band shows the extent of the error in the best-fit EXAFS amplitude.

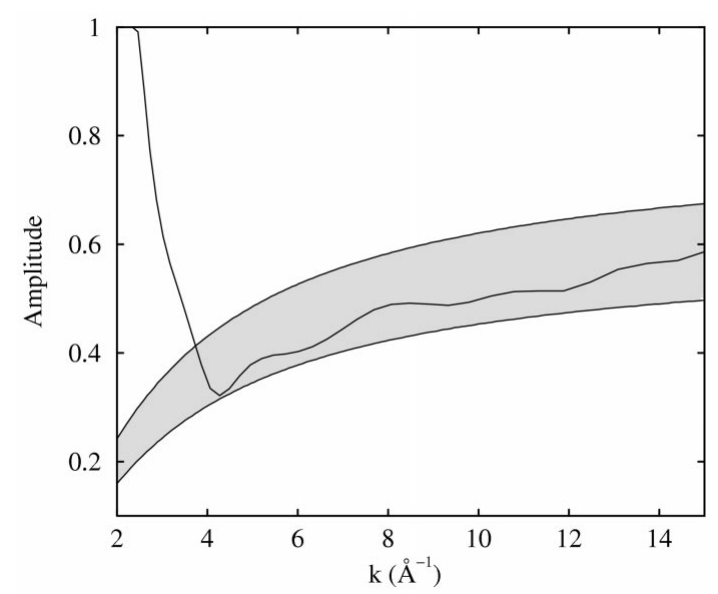

Figure 6

Calculated and best-fit EXAFS reduction factors plotted against photoelectron wavevector for copper. The black line gives the calculated result, the solid band shows the extent of the error in the best-fit EXAFS amplitude. index of 37.6. In both fits the default EXCURV value for the corehole lifetime of $0.43 \mathrm{eV}$ was used, whilst the experimental resolution of $\sim 0.1 \mathrm{eV}$ was considered small enough to be neglected.

The experiment and theory diverge at low energies where multiple scattering becomes more important, but between approximately 3 and $14 \AA^{-1}$ both theoretical approaches give reasonable fits to experiment. It is noticeable that at low $k$ the $\mathrm{X} \alpha$ potential result is killed by the correction factors whilst the HL potential gives much larger magnitudes and a much more satisfactory match to experiment. In this region the ad hoc correction factors applied to the EXAFS amplitudes in the $\mathrm{X} \alpha$ calculation greatly overestimate the inelastic losses. This accounts for the better fit index obtained using the HL potential.

Fig. 4(c) compares the two theoretical calculations described above to a third calculation performed using the HL potential without any amplitude corrections applied. This succinctly shows the problem with all real potential calculations of the EXAFS: namely amplitudes which are much too large.

Finally, in Fig. 4(d), we demonstrate the accuracy of the approximate result from equation (18) in estimating the EXAFS amplitudes in the presence of an imaginary potential [equation (18)]. The solid line shows the calculated amplitude from equation (18) whilst the circles are obtained by dividing the amplitudes given by the HL potential with those found using the $\mathrm{X} \alpha$ potential with no corrections. These two calculations give results for the fine structure which are slightly out of phase, therefore, to obtain a rough estimate of the effect of the imaginary part of the HL potential on the amplitude of an exact calculation we have compared the results of the two calculations at corresponding peaks. The circles are the ratios of these amplitudes at successive peaks, whilst the crosses show the corresponding ratio of peak heights between the lossless $\mathrm{X} \alpha$ calculation and the $\mathrm{X} \alpha$ calculation with added correction factors. The differing real parts of the HL and X $\alpha$ potentials give slightly different scattering strengths for each of the shells which further complicates the situation, but we have ignored this effect in these plots. From the plots it is apparent that all three sets of amplitudes are roughly consistent in the region primarily of interest to EXAFS data analysis, whilst the amplitudes given by the ad hoc correction factors to the $\mathrm{X} \alpha$ potential appear to be tending to zero at low energies.

Figs. 5, 6 and 7 show the major results of this paper. We plot the EXAFS amplitude as a function of photoelectron wavevector in

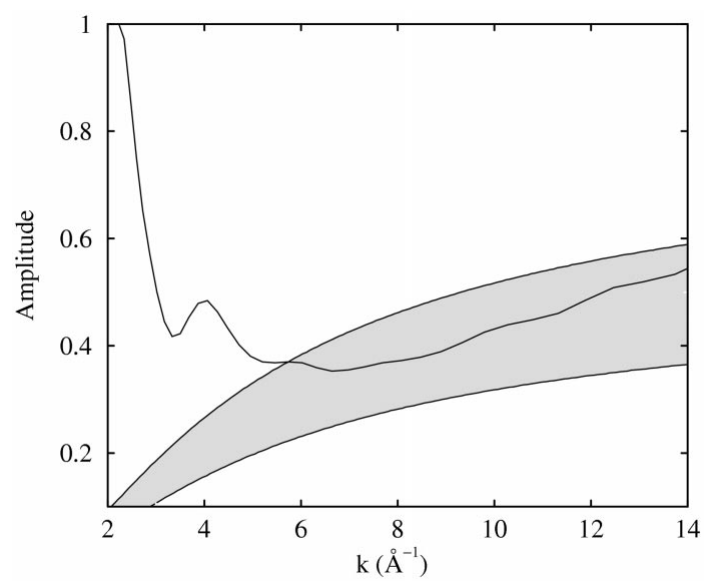

Figure 7

Calculated and best fit EXAFS reduction factors plotted against photoelectron wavevector for silver. The black line gives the calculated result, the solid band shows the extent of the error in the best-fit EXAFS amplitude. 
inverse Angströms. The origin of the plots has been set at $k=2 \AA^{-1}$, approximately at the position of the edge. The photoelectron wavevector is not zero at the $\mathrm{X}$-ray absorption edge because of the finite Fermi energy.

The EXAFS amplitude is calculated using equation (18) for three very different elements: silicon, copper and silver. This is then compared with known values of the empirical parameters, $s_{o}^{2}(k)$ and $e^{-2 r_{j} / \lambda(k)}$, fitted using the real X $\alpha$ potential for the three elements with the data analysis program EXCURV 98 , in the same way as described previously for silicon. The best-fit values of $s_{o}^{2}$ and the effective constant imaginary potential, $V_{P I}$, were found to be (Gurman, 1999) $s_{o}^{2}=0.70 \pm 0.1$ for copper and $s_{o}^{2}=0.67 \pm 0.15$ for silver. The $V_{P I}$ values used were: $-4.0 \mathrm{eV} \pm 0.2 \mathrm{eV}$ for copper and $-6.3 \mathrm{eV} \pm 0.2 \mathrm{eV}$ for silver where the quoted uncertainties are $2 \sigma$ errors. In the figures the solid band shows the product of the fitted vales of $s_{o}^{2}$ and $e^{-2 r_{j} / \lambda(k)}$ within the limit of experimental error whilst the black line gives the calculated EXAFS amplitude [equation (18)]. Below a $k$ of about $3 \AA^{-1}$ the historically used EXAFS reduction parameters exhibit unphysical behaviour. Using a constant imaginary part to the potential we obtain a zero mean free path at low $k$ and hence an EXAFS amplitude of zero. This is obviously incorrect as, below, the minimum electron excitation energy in the system there cannot be any inelastic scatterings and hence the EXAFS amplitudes should be unity. This was a known problem in older data analysis.

At low X-ray energies above the edge the calculated amplitude using the HL potential is unity. This is correct. At low energies the photoelectron is insufficiently energetic to excite a plasmon anywhere in the atomic muffin tin. The energy at which the amplitude begins to deviate from unity corresponds to the plasmon frequency in the region of low electronic density toward the edge of the muffin tin. In an atomic calculation this cut-in energy would be that of the most weakly bound atomic orbital. The rapid cut-in of the amplitude reduction is a feature of the HL potential. This occurs because the majority of the imaginary part of the HL potential comes from the region of almost constant electronic density toward the edge of the muffin tin spheres.

At high $k$ the HL potential overestimates the EXAFS amplitudes. The HL potential models the extrinsic loss effects and so, like the mean-free-path term, it will disappear at high energies. From Figs. 5, 6 and 7 it is clear that the calculated amplitudes are still increasing at $k=15 \AA^{-1}$. The HL potential itself varies as $1 / k$ at large $k$, and, as the radial wavefunctions, $R_{l}(k r)$, are also proportional to $1 / k$ at high energies, the EXAFS amplitudes given by the HL potential will obviously tend to unity. However, using the sudden approximation we can show that the intrinsic loss parameter, and hence the total amplitude reduction, should tend to a constant value of about 0.7 at high photoelectron energies (Rehr et al., 1978; Roy \& Gurman, 1997). The difference arises because the HL potential does not include a contribution from the core hole.

The losses obtained from the HL potential agree reasonably well with the total reduction given by the semi-empirical reduction parameters. The HL potential must therefore overestimate the losses produced by the mean-free-path effects alone. Over the range of a typical EXAFS spectrum, 3 to $14 \AA^{-1}$, the calculated reduction parameters lie within the error range of the best-fit experimental data. The correlation between the calculations and the fitted empirical parameters can be measured in this region using the $R$-factor,

$$
R=\frac{\sum\left|A_{\text {expt }}-A_{\text {theory }}\right|}{\sum\left|A_{\text {expt }}\right|} \times 100 \%
$$

We find $R=4.9 \%$ for silicon, $R=6.5 \%$ for copper and $R=10.4 \%$ for silver. These uncertainties are much lower than those observed in the best fit parameters for the various elements: $15.5 \%$ for silicon, $16.1 \%$ for copper and $24.4 \%$ for silver, which suggests that the reduction given by the HL potential is equivalent to that produced by the semiempirical reduction parameters.

EXCURV 98 was also used to fit values for the adjustable parameter, $s_{o}^{2}$, using the HL potential. We found values of $s_{o}^{2}(k)=$ $0.92 \pm 0.10$ for silicon, $s_{o}^{2}(k)=1.05 \pm 0.05$ for copper and $s_{o}^{2}(k)=$ $1.02 \pm 0.05$ for silver. These values are all consistent with unity which suggests that additional amplitude fitting parameters should not be used when data fitting using the HL potential. It would appear that the HL potential gives extremely good results for EXAFS calculations, albeit accidentally.

Fitting the amplitude reduction parameter for a number of elements we obtain the points shown in Fig. 8. These points exhibit some scatter but are generally much larger than the values of $s_{o}^{2}$ which we would expect to obtain using the $X \alpha$ potential and a mean-freepath term. We therefore conclude that the HL potential significantly overestimates the extrinsic losses to the EXAFS amplitude. Empirically, the HL potential includes most, if not all, of the contribution from the intrinsic loss events.

We believe this conclusion may be tentatively generalized to molecular and ionic compounds. Fig. 8 demonstrates that the HL potential gives good results for a number of different atomic systems, whilst the general experience of EXAFS users is that this potential gives reasonable amplitudes for a range of different compounds: hence the success of the major programs FEFF and EXCURV which use the HL potential. Tyson et al. (1992) find good EXAFS amplitudes using the HL potential with no corrections for intrinsic effects for the molecular systems $\mathrm{SF}_{6}, \mathrm{GeCl}_{4}$ and $\mathrm{Br}_{2}$, whilst Amiss \& Gurman (1999) come to the same conclusion for a biological system. To study in detail the effect of anisotropic charge densities we would have to move beyond the muffin tin approximation. Schemes for full potential calculations have been proposed (see, for example, Natoli et al., 1986) but the calculations by Tyson et al. (1992) suggest that the differences between a full potential treatment and the muffin tin approximation are small.

To summarize, the HL potential was designed to model the extrinsic loss effects. However, using equation (18) or the Daresbury program EXCURV98 we find that the imaginary part of the HL

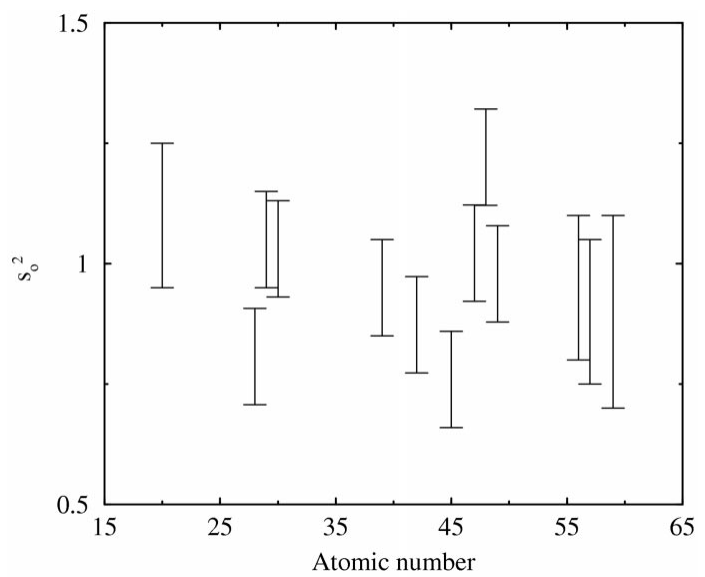

Figure 8

The amplitude reduction factor obtained by fitting EXAFS spectra using the HL potential with the Daresbury program EXCURV98. Plotted as a function of atomic number. 
potential significantly overestimates the losses produced by the extrinsic effects alone. Instead it seems to, qualitatively, reproduce the total losses to the EXAFS amplitude even though the average imaginary part is consistent with the constant potential of $-4 \mathrm{eV}$ obtained from LEED experiments. The reason for this is the shape of the imaginary part of the HL potential. The average value would correctly account for the extrinsic losses; however, the HL potential emphasizes the middle and outer regions of the muffin tin as opposed to the region near to the nucleus where $r^{2} R_{l}(r)$ is small anyway and therefore overestimates the losses. This can be immediately seen from a calculation of the energy-dependent mean free path obtained from the HL potential.

In Fig. 9 we plot the mean free paths for aluminium obtained from three different calculations and compare to data reproduced from Penn (1987). In an isolated atom the loss of photoelectron flux is related to the mean free path by $\exp \left[-r_{m t} / \lambda(k)\right]$ where $\lambda$ is the relevant mean free path for flux. Equating this expression to the flux loss caused by the imaginary part of the HL potential we can immediately obtain an expression for the mean free path given by the HL potential,

$$
\lambda_{H L}(k)=\frac{-r_{m t}}{\log \left(1+k\left\langle R_{l}\left|V_{I}\right| R_{l}\right\rangle\right)} .
$$

In terms of a constant imaginary potential the flux mean free path can be written $\lambda(k)=k / 2 V_{P I}$.

In Fig. 9 we can see that the HL potential gives a mean free path that is much shorter than that found experimentally. The average part of the HL potential, however, reproduces the experimental results to much better accuracy and gives magnitudes for the mean free path comparable with those obtained from a constant imaginary potential of $-4 \mathrm{eV}$. Thus the HL potential will give good results for free electrons injected into the material which see the average potential. The mean free path for both the HL potential and the average HL potential goes to infinity below the minimum plasmon excitation energy where no inelastic scattering events are possible.

To emphasize this result, the radially dependent HL potential is incorrect. It predicts values for the mean free path of internally produced photoelectrons which are much too small. The average HL potential, on the other hand, gives values which agree tolerably well

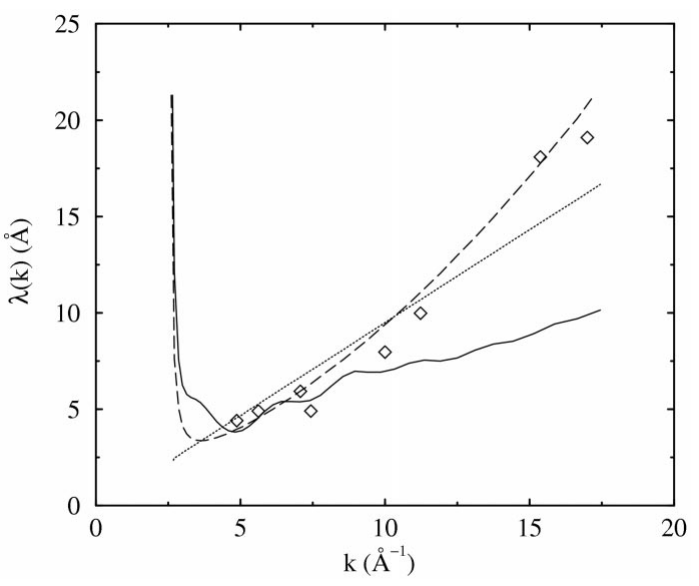

Figure 9

The energy-dependent mean free path in $\AA$ plotted against $k$ in $\AA^{-1}$ for aluminium. The solid line is that obtained from the imaginary part of the HL potential. The dashed line is calculated from the average imaginary part of the HL potential, the dotted line is that given by a constant imaginary potential of $-4 \mathrm{eV}$ and the diamonds are data reproduced from Penn (1987). with experiment. It is the constant density, $r$ independent, imaginary part of the HL potential (equivalent to the average HL potential) that, for example, Rehr \& Albers (2000) have claimed to give good agreement with the experimentally found mean free paths.

Extensions to the HL potential incorporating a proper treatment of the core-hole and multiple electron excitations, have been proposed [see, for example, Fujikawa et al. (2000); Natoli et al. (1990)], and have even been used for data analysis (Wu et al., 1996). However, the implementation of these methods is still far from routine and we are unaware of any practical alternatives to the use of the HL potential for data fitting at present.

\section{Conclusion}

In this paper we have presented a systematic study of the effect of the imaginary part of the Hedin-Lundqvist exchange and correlation potential on the calculated EXAFS amplitude. An expression has been derived whereby the amplitudes given by an imaginary potential may be evaluated independently of the full EXAFS function. These amplitudes are then compared with fitted values for the EXAFS amplitude reduction parameters obtained from experimental data using EXCURV 98.

We find that the HL potential overestimates the losses to the EXAFS due to the finite photoelectron mean free path alone. However, it happens to give excellent agreement with the total experimental losses in the energy range of most EXAFS data analysis.

Empirically the HL potential seems to account for all of the losses to the EXAFS amplitudes. It should therefore not be necessary to use the amplitude fitting parameters $s_{o}^{2}$ and $V_{P I}$ when data fitting using this potential.

However, the HL potential is 'merely a phenomenological model that happens to work for the EXAFS' (Tyson, 1991); by no means does it rigorously describe all the processes contributing to the inelastic scattering of the photoelectron. Effectively the HL potential underestimates the mean free path of the photoelectron leading to some ad hoc inclusion of the intrinsic effects. It is therefore possible that some further fitting of the amplitude using the variable parameters will be necessary when using the HL potential for EXAFS calculations.

\section{References}

Amiss, J. \& Gurman, S. J. (1999). J. Synchrotron Rad. 63, 387-388.

Binsted, N. (1998). EXCURV98. Computer Program for EXAFS Data Analysis. CLRC Daresbury Laboratory. (http://srs.dl.ac.uk/xrs/Computing/ Programs/excurv97/e61.htm.)

Bransden, B. H. \& Joachain, C. J. (1983). Physics of Atoms and Molecules, p. 494. Longman Scientific and Technical.

Fujikawa, T., Hatada, K. \& Hedin, L. (2000). Phys. Rev. B, 62, 5387-5398.

Gurman, S. J. (1983). J. Phys. C: Solid State Phys., 16, 2987-3000.

Gurman, S. J. (1999). Personal communication.

Gurman, S. J., Binsted, N. \& Ross, I. (1984). J. Phys. C: Solid State Phys. 17, $143-151$.

Gurman, S. J. \& McGreevy, R. L. (1990). J. Phys. Condens. Matter, 48, $9463-$ 9473.

Hedin, L. \& Lundqvist, S. (1969). Solid State Phys. 23, 2-181.

Lee, P. A. \& Beni, G. (1976). Phys. Rev. B, 15, 2862-2883.

Lee, P. A. \& Pendry, J. B. (1975). Phys. Rev. B, 11, 2785-2811.

Natoli, C. R., Benfatto, M., Brouder, C., Ruiz Lopez, M. F. \& Foulis, D. L. (1990). Phys. Rev. B, 42, 1944-1968.

Natoli, C. R., Benfatto, M. \& Doniach, S. (1986). Phys. Rev. B, 34, 4682-4694. Newville, M., Livins, P. \& Yacoby, Y. (1993). Phys. Rev. B, 43, 14126-14131. 


\section{research papers}

Pendry, J. B. (1974). Low Energy Electron Diffraction. London: Academic Press.

Penn, D. R. (1987). Phys. Rev. B, 35, 482-486.

Rehr, J. J. \& Albers, R. C. (2000). Rev. Mod. Phys. 72, 621-654.

Rehr, J. J., Stern, E. A., Martin, R. L. \& Davidson, E. A. (1978). Phys. Rev. B, 17, 560-565.

Roy, M. \& Gurman, S. J. (1997). J. Phys. IV France Colloq. C2, 151-152.

Roy, M. \& Gurman, S. J. (1999). J. Synchrotron Rad. 6, 228-230.
Roy, M., Lindsay, J., Louch, S. \& Gurman, S. J. (2001). J. Synchrotron Rad. 8, 1103-1108.

Tyson, T. A. (1991). PhD thesis, Stanford University, USA.

Tyson, T. A., Hodgson, K. O., Natoli, C. R. \& Benfatto, M. (1992). Phys. Rev. $B, \mathbf{4 2}, 5997-6019$.

Wu, Z., Benfatto, M. \& Natoli, C. R. (1996). Phys. Rev. B, 54, 13409-13412. Zabinsky, S. I., Rehr, J. J., Ankudinov, A. L., Albers, R. C. \& Eller, M. (1995). Phys. Rev. B, 52, 2995-3009. http://leonardo.Phys.washington.edu/feff/ 